\title{
The Effect of Iodine Based Products on Unicellular Algae from Genus Prototheca
}

\author{
Sorin RĂPUNTEAN *, Gheorghe RĂPUNTEAN, Flore CHIRILĂ, Nicodim Iosif FIȚ, George Cosmin NADǍŞ \\ University of Agricultural Sciences and Veterinary Medicine Cluj-Napoca, Faculty of Veterinary Medicine, \\ 3-5 Mănăștur Street, 400372, Romania \\ *Corresponding author: sorin.rapuntean@gmail.com
}

Bulletin UASVM Veterinary Medicine 72(2) / 2015,

Print ISSN 1843-5270; Electronic ISSN 1843-5378

DOI:10.15835/buasvmcn-vm: 11439

\begin{abstract}
Iodine based products have been and are still used in medicine as disinfectant or antiseptic substances, because of their bactericidal, sporicidal, protocidal and disinfection effect. Algaecide effect has been less studied and represents a strong motivation for this study.

This paper is aiming to test the sensitivity to iodine based products of unicellular algae of the genus Prototheca. Such products might be used for the treatment of diseases involving these pathogens.

A total number of twenty-two Prototheca zopfii strains isolated from cows with mastitis or bovine shelters, were tested within this study. The algal strains were identified based on morphological characteristics (shape, size, presence of endospores), cultural (liquid and solid media characteristics) and biochemical (fermentation of sugars). Prototheca wickerhamii ATCC 16529 reference strain was also included in the evaluation. Iodinated products used were represented by: Lugol solution, iodine tincture, betadine, videne and potassium iodide. Determination of the inhibitory effect was measured by diffusion technique in agarose gel and by liquid medium dilution method. For the tincture of iodine and betadine, the inhibitory effect was also appreciated in relation with the time of contact $(5,10,15,30$ and 60 minutes).

By the agar diffusion technique, it was found that inhibition zones varying sizes were correlated with the composition of those products. For the 22 Prototheca zopfii tested strains, the size of inhibition areas was within the following values (average diameter): iodine tincture $25.71 \mathrm{~mm}$, betadine $26.4 \mathrm{~mm}$, videne $25.61 \mathrm{~mm}$, Lugol's solution $11.33 \mathrm{~mm}$ and potassium iodide $11.14 \mathrm{~mm}$. For Prototheca wickerhamii strain inhibition areas had the following values: betadine $26 \mathrm{~mm}$, iodine tincture $24 \mathrm{~mm}$, videne $24 \mathrm{~mm}$, Lugol's solution $10 \mathrm{~mm}$, potassium iodine $14 \mathrm{~mm}$. In case of different contact times meant to establish the algaecide effect - occurred in the first 5 minutes of contact, for both iodine tincture and betadine.

Iodine based products tested showed inhibitory effect on algal strains. The best efficacy was found for betadine and iodine tincture (pharmaceutical/commercial products). The inhibitory effect occurs in the first 5 minutes of contact. There was no resistance phenomenon nor the presence of resistant colonies in the inhibition zones.
\end{abstract}

Keywords: iodine based products, Prototheca, unicellular algae

\section{INTRODUCTION}

Because of its chemical properties, iodine is used in various fields of science and technology, including medicine. It can be used as the self, or as $\mathrm{I}_{3}$ anion dissolved in water. Examples of such preparations are: iodine tincture (iodine in ethanol or iodine and sodium iodide in a mixture of ethanol and water), Lugol's solution (iodine and potassium iodide in water). Because iodine is only slightly soluble in water, the solutions are prepared in the form of dyes in ethanol. Iodoform, iodclorhidroxichinolina, iodophors, sodium iodide and potassium, exercise also bactericidal effects by releasing iodine [25]. Iodine can come from 
Iodophors containing iodine complexed with a surfactant and solubilizing agent, povidone iodine is popular (polyvinilpyrolidon and iodine) and iodine cadexomer (polysaccharide complex and iodine) (Cooper, 2004; Bootham, 2009; Sibbald et al., 2011).

Since the phenomenon of antibiotic resistance has spread and amplified in recent years, alternatives for finding other preparations which exhibit antimicrobial effects have been frequently approached. Among them preparations extracted from plants or based on different chemical elements ( $\mathrm{Ag}, \mathrm{Cu}, \mathrm{Au}, \mathrm{Zn}, \mathrm{Fe}, \mathrm{I}$ and others). In the case of iodine and iodine-based preparations there are mentions that the resistance phenomenon is not installed or it is exceptionally uncommon (Boothman, 2009).

Iodine and iodine based products were processed and used as a disinfectant or antiseptic in medicine, especially in the preparation of the operative field before surgery, known to have bactericidal, sporicidal and virulicid activity. The effect is especially microbicide against bacteria and viruses, but less intense against some forms of parasitic cysts such as Giardia and Criptosporium [26]. Algaecide effect has been less studied, good reason for the research within this study.

Unicellular algae of the genus Prototheca began to be increasingly popular, with implications causing fatal chronic granulomatous lesions localized in different tissues. Illness are seen more frequently in cows (as mastitis) in dogs and several other species of warm-blooded and poikilothermic animals but humans too. It is known that the treatment with antibiotics and chemotherapeutics is frequently ineffective, animal slaughter or euthanasia is in some cases the final solution (Buyukmihci et al., 1975). Contaminated milk is a major risk to public health (Krukowski et al., 2013). Algae are considered as opportunistic pathogens with a low virulence, but can also indicate the pathogenicity of the host for various forms of immunosuppression such aspects being found in natural (Woolorich et al., 1994; Wirth et al., 1999) and experimental infections (Răpuntean et al., 2001).

The paper aimed to test the in vitro effectiveness of iodine based products against 22 Prototheca zopfii strains isolated from bovine mastitis cow milk or in bovine shelters and ATCC 16529 Prototheca wickerhamii strain. The strains were isolated for several years (2008-2014), and have been preserved in the collection of Microbiology Department, Faculty of Veterinary Medicine from Cluj-Napoca. The examination also covered a standardized strain: Prototheca wickerhamii ATCC 16529.

\section{MATERIALS AND METHODS}

We tested 22 Prototheca zopfii strains isolated from milk from cows with mastitis or cattle sheds. Prototheca strains have been identified based on several characteristics: morphological (shape, size, presence of endospores - for which the size and arrangement constitutes an important criterion for identification), cultural (growth on glucose culture medium, liquid and solid, incubated at $37^{\circ} \mathrm{C}$; the colonies were small initially, gradually increasing in size, reaching 3-4 $\mathrm{mm}$, white, matte, with slightly lobed edges and grainy/cauliflowerlike surface) and biochemical (fermenting glucose, glycerol and fructose, variable at other sugars; oxidase and catalase positive reaction; indole and $\mathrm{H}_{2} \mathrm{~S}$ negative). Strains are preserved in soft agar. Iodinated products testing were: Lugol, iodine (SC Hippocrates SRL, Bucharest), Betadine ${ }^{\circledR}$ (EGIS Pharmaceuticals PLC, Budapest Hungary), Videne $^{\circledR}$ (Adams, Gorforth Leeds, England) and $10 \%$ potassium iodide (GL Pharma $\mathrm{GmbH}$ ).

The work technique was based on the 2013 CLSI vet. method (26) with some modifications listed below.

The inhibitory effect established by disc diffusion method. The tested strains were grown in glucose broth and incubated at $37^{\circ} \mathrm{C}$ for 48 hours. The prepared suspension was used to flood $9 \mathrm{~cm}$ diameter Petri dishes containing glucose agar having a thickness of $3 \mathrm{~mm}$. The suspension was evenly spread using a Drigalski spatula, then the excess liquid removed. The plates were placed in thermostat for 30 minutes with the lid half open. After this time, in the agarose gel, wells were cut out using a $4 \mathrm{~mm}$ diameter mold, according to a radial pattern. This size, considering the thickness of 3 $\mathrm{mm}$ of the agar layer enables a well with a narrow diameter, which ensures a good distribution of the iodinated product in the surrounding gel. The wells were filled with $20 \mu \mathrm{l}$ of the tested medium. The plates were placed in a thermostat at $37^{\circ} \mathrm{C}$ and examined at 24 and 48 hours.

The inhibition areas were appreciated, measuring their diameter (mm). To establish the 
algaecide effect, inoculations were performed on glucose broth.

In a different working method, from a thick suspension, prepared from Prototheca colonies developed on the agar, dilutions were performed in saline to densities according to McFarland standards: $0.5 ; 1 ; 2 ; 3 ; 4$ and 5 . Each dilution was used to inoculate two Petri dishes ( $1 \mathrm{ml}$ per plate). After homogenization and removal of excess, the plates were placed in a thermostat for the surface to dry (30 minutes), then cut wells with a diameter of $4 \mathrm{~mm}$ filled with $20 \mu \mathrm{l}$ of each iodinated product. The plates were incubated in a thermostat at $37^{\circ} \mathrm{C}$ and examined at 24 and 48 hours.

Establishing the efficiency of iodized products (tincture of iodine and Betadine) by dilution method. A quantity of $4.5 \mathrm{ml}$ of glucose broth was distributed in a series of five tubes. To the first tube was added $0.5 \mathrm{ml}$ of the iodinated product which, after mixing, was passed in succession 0.5 $\mathrm{ml}$ in the following tubes, in the end removing $0.5 \mathrm{ml}$ of the tube no. 5. The resulted iodinated product dilution rate $\mathrm{x} 10$ is $\left(10^{-1}, 10^{-2}, 10^{-3}, 10^{-4}\right.$, and $\left.10^{-5}\right)$. Subsequently, each tube was inoculated with a fixed amount of $5 \mu \mathrm{l}(\sim 2100 \mathrm{UFC} / 1 \mathrm{ml})$ of Prototheca zopfii (strain 22) and Prototheca wickerhamii (ATCC 16529). Control tubes, without iodized products: 1) tube that allows the development of culture (positive control); 2) non-inoculated tube to check the culture medium (negative control).

The tubes were incubated at $37^{\circ} \mathrm{C}$ and maintained for 24 hours. To verify the inhibitory effect of each tube in the dilution series set, glucose agar plates were streaked using one plate for each tested strain, and the plates were divided into 5 sectors of a triangular shape corresponding to each dilution. The plates were incubated at $37^{\circ} \mathrm{C}$ and examined at 24 and 48 hours, but kept under observation a further 5 days. The control tubes checking was done separately on another glucose agar plate.

Evaluation of the inhibitory at different contact times. The tested strains were Prototheca zopfii (22 strain) and Prototheca wickerhamii (ATCC 16529), and the testing was done to the iodine tincture and Betadine. In a 10/110 mm tube, 0.9 $\mathrm{ml}$ iodine tincture or Betadine was dispensed, followed by $0.1 \mathrm{ml}$ culture two strains tested in glucose broth. Contact times were 5', 10', 15', 30 ' and 60'. After the end of each contact time streaking's were performed on solid medium (glucose agar), previously distributed in Petri dishes, and divided into 5 triangular sectors. The plates were incubated in a thermostat at $37^{\circ} \mathrm{C}$. The interpretation was made at 24 and 48 hours, with the final examination after 5 days.

\section{RESULTS AND DISCUSSION}

The results for disc diffusion method test. Iodine based products revealed inhibitory effect against Prototheca strains. Inhibition areas were correlated with products composition and the ability of iodine release.

For the 22 Prototheca zopfii tested strains, the average size of inhibition areas were as following: Betadine 16 to $30 \mathrm{~mm}$ (26.4), tincture of iodine 20 to $30 \mathrm{~mm}$ (25.71), Videne 16 to $30 \mathrm{~mm}$ (25.61), Lugol's solution 10 to14 $\mathrm{mm}(11,33)$ and potassium iodide 10 to $24 \mathrm{~mm}(11,14)$ (Fig. 1).

For Prototheca wickerhamii strain, the inhibition areas were the following: Betadine 26 $\mathrm{mm}$, tincture of iodine $24 \mathrm{~mm}$, Videne $24 \mathrm{~mm}$, Lugol's solution $10 \mathrm{~mm}$, potassium iodide $14 \mathrm{~mm}$ (Fig. 2). There was no presence of drug resistant or resistant colonies in the inhibition zones. The inoculations carried out from the inhibition zones in glucose broth tubes remained sterile, confirming that the induced effect was algaecide.

The results obtained at the test of different inoculum density according to McFarland standards. The aim of this test was to evaluate whether the inoculum density is influencing the size of the inhibition areas. Two strains were initially tested, Prototheca zopfii (strain 22) and Prototheca wickerhamii (ATCC collection strain).

It was found that the size of the inhibition zones has been less affected by the density of the inoculum, the recorded differences being of 2-4 $\mathrm{mm}$ between the smaller densities ( 0.5 to 1 ) and the larger (2, 3, 4 and 5) (Tab. 1, P. zopfii strain 22).

The results were similar for $P$. wickerhamii strain, as well as other 5 strains of $P$. zopfii subsequently tested.

Pictures with the inhibition areas obtained for the plates with the inoculum of different densities are observed below Fig. 3 ( $a$ and $b$ ).

The results obtained for diluted iodinated product. Algaecide type inhibitory effect was found only in $10^{-1}$ dilution. In that dilution tube environment it remained clear, with a light purple colour (due to iodine). The other dilutions/tubes, 
the medium also remained clear, for the first 24 hours, with the presence of reduced sediment, which easily disperse when stirring. During the examination at 48 hours was found a thin pellicle on the surface and the deposit became more abundant, meaning that algae multiplied.

The inoculations on glucose agar plates made from each dilution (after 48 hours) revealed that only $10^{-1}$ dilution have not developed colonies (algaecide effect). On the other dilutions (10 2 to $10^{-5}$ ) Prototheca typical colony developed; at 24 hours they were small (less than $0.5 \mathrm{~mm}$ in diameter), circular, opaque, white, at 48$72 \mathrm{~h}$, they grow in size $(2-4 \mathrm{~mm})$, having an irregular surface, the edges slightly lobed, white. A good characterization of colonies can be done by examining using the magnifying glass, where typical structure and other algae details (cauliflower surface aspect) were revealed and can differentiate in the situation of contamination by other bacteria colonies mainly staphylococci, micrococci and yeast.

When examining the wet smears (dispersion in a drop of Lügol), made of tubes that Prototheca dilutions remained viable $\left(10^{-2}\right.$ to $\left.10^{-5}\right)$, it was found that the cells have undergone morphological changes, which reflected in the growth and formation of typical colonies on solid culture media. This behaviour was seen in both tested strains ( $P$. zopfii and $P$. wickerhamii), both for iodine tincture and Betadine (Fig. 4 ( $a$ and b), Fig. 5 ( $a$ and $b)$ ). This means that at higher dilutions of $10^{-1}$, algaecide effect is no longer achieved due to dilution of the iodide product resulting in subinhibitory values.

Such results reveal that in order to obtain good efficiency in prevention or treatment of protothecosis, iodine based products must not be diluted more than $10^{-1}$.

The inhibitory effect observed at different contact times. When examining the inoculated plates (glucose agar) after each contact time - 5, $10,15,30$ and 60 minutes, it was observed that no colonies have developed in any of the tested strains after incubation at $37^{\circ} \mathrm{C}$ for 24 and 48 hours (Fig. 6 and 7). Algaecide effect manifested itself in the first 5 minutes of contact, for both iodine tincture and Betadine. The effect was algaecide, because the microorganisms presented no growth in culture plates even if maintaining the plates for the following 5 days at laboratory temperature.

The inoculations made in glucose broth from the areas corresponding to each contact time, and examined at 24 and 48 hours, remained sterile, confirming the induction of inhibitory effect. The experiment was repeated for another 5 strains, but the result was the same - total inhibition. The researches revealed that the iodine based products (Betadine and iodine tincture), have an inhibitory effect - algaecide, which is observed within the first five minutes of contact with the products. The choice of 5 minutes time as first stage of contact between the iodide products and a suspension of prothotecs, has been based on the comparison of the sensitivity of bacteria, which are usually inactivated in 15-30 seconds (according to

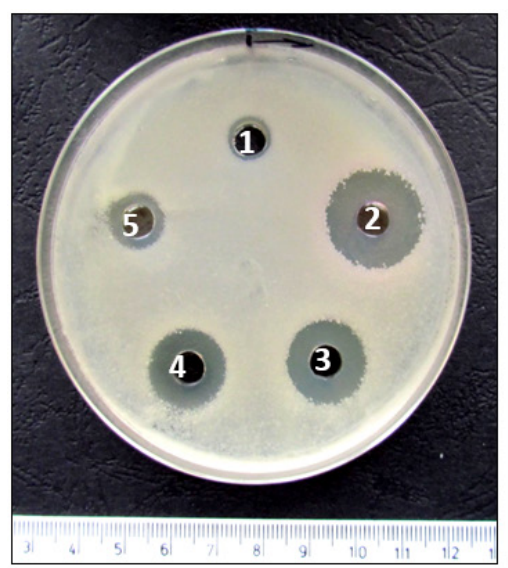

Fig. 1. Inhibition areas, Prototheca zopfii (strain 22)

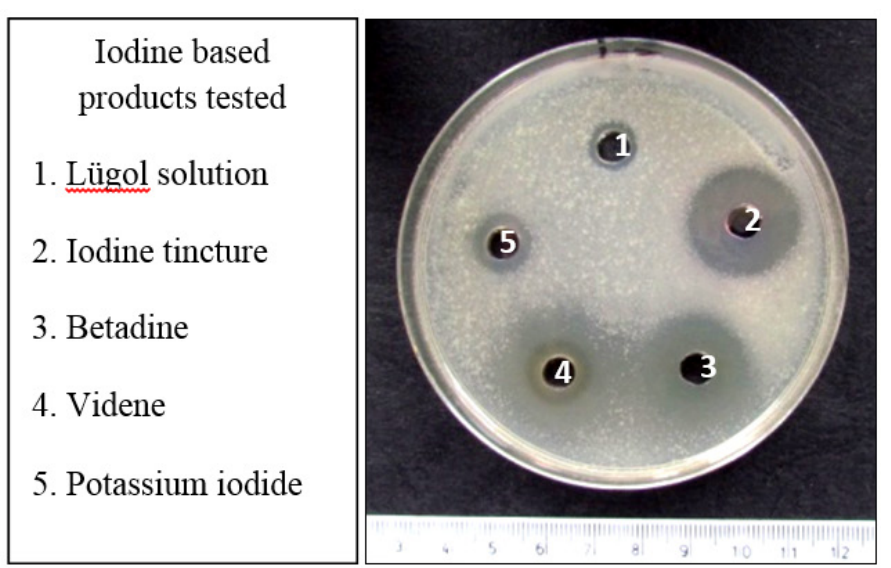

Fig. 2. Inhibition areas, Prototheca wickerhamii (16529 ATCC) 
the literature) (23). Knowing that prototecile are larger and have thicker cell wall, we considered that five minutes can be taken as the minimum time necessary to manifest inhibitory effect. In literature, we found no information in this regard.

\section{DISCUSSION}

The antimicrobial properties of iodine are known for long time, but research on the inhibitory effect of iodine and/or various iodine based products were amplified as a result of the increasing expansion of the phenomenon of antibiotic resistance. Numerous studies confirmed that iodine based products have bactericidal, sporicidal, cisticid and virulicid effect. Iodine exact mode of action is not fully known, but it is complex (Cooper, 2004; Bootham, 2009; Sibbald et al., 2011).

It is believed that the iodine based products are able to quickly penetrate the cell wall of different microorganisms, causing structural and functional changes of the cell membranes and the nucleic acids by denaturation of proteins and enzymes, which results in rapid death of various microorganisms (Gottardi, 1999; Bootham, 2009; Sibbald et al., 2011). The activity is immediately, but also over time due to persistence. The efficacy and stability of the aqueous solutions is influenced by the values of pH (Gottardi, 1999).

A broad use of these preparations is mentioned in the treatment of various types of wounds in which the antibiotic-resistant bacteria frequently colonized (Giacometti et al., 2002). Direct application on wounds caused some controversy about possible toxic effects on cells (Burck, 1988; Drosou et al., 2003). It is believed that cytotoxicity effects are reduced and occur only in the event of prolonged treatments (Sibbald et al., 2011). On the other hand, it is shown that the iodine preparations (povidone iodine and cadexomer iodine) reduced the bacterial load, which promotes epithelial regeneration and healing (McLure et al., 1992; Lamme et al., 1998; Mertz et al., 1999).

Some products, such as iodins may even penetrate biofilms, which are formed on different surfaces, including wounds (Bootham, 2009; Phillips et al., 2010). In vitro, microbicide effect has been demonstrated by various study methodologies. It shows that povidone iodine causes deadly alterations $>99 \%$ of microbial cells (methicillin susceptible and resistant Staphylococcus aureus strains) within 10 seconds of exposure (Lacey et al., 1993). Iodine is active

Tab. 1. The size of inhibition areas at different densities of the inoculum

\begin{tabular}{cccccc}
\hline & \multicolumn{5}{c}{ Inhibition areas (ø mm), strain 22 } \\
\cline { 2 - 6 } Density & \multicolumn{2}{c}{$\begin{array}{c}\text { Iodine } \\
\text { Lugol sol. }\end{array}$} & Betadine Videne & $\begin{array}{c}\text { Potassium } \\
\text { tincture }\end{array}$ \\
\hline 0,5 & 10 & 24 & 20 & 20 & 12 \\
\hline 1 & 10 & 22 & 20 & 20 & 12 \\
\hline 2 & 10 & 22 & 18 & 18 & 12 \\
\hline 3 & 8 & 22 & 18 & 18 & 10 \\
\hline 4 & 8 & 22 & 18 & 16 & 10 \\
\hline 5 & 6 & 22 & 16 & 16 & 10 \\
\hline
\end{tabular}

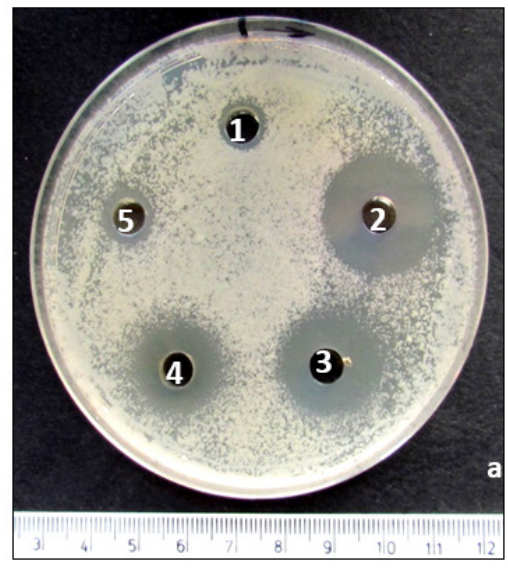

3a) Inhibition areas at density 0,5

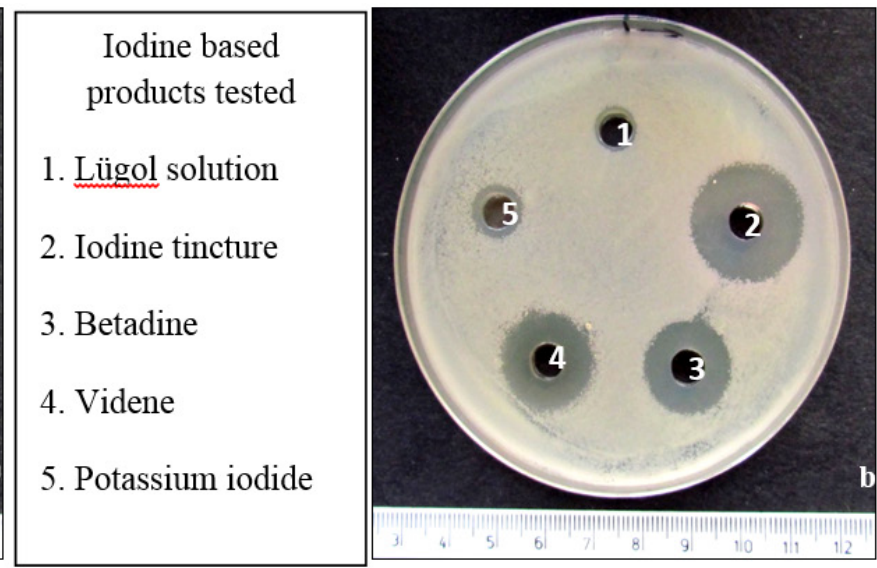

3b) Inhibition areas at density 5

Fig. 3. Inhibition areas at different inoculum densities (P. zopfii strain 22) 
against many pathogenic bacteria, most being killed in 15-30 seconds of contact [24].

It is important to note that iodine preparations do not develop resistance phenomena. However, in the case of $S$. aureus strains isolated from mastitic cow milk, a variation of the iodine minimum inhibitory concentration was described (Azizoglu et al., 2013), and even the induction of in vitro resistance by repeated passages on culture media containing subinhibitory doses of antimicrobials (El Behiry et al., 2012).

Unicellular algae of the genus Prototheca have become increasingly involved in the production of human and animal diseases, in both warm-blooded and cold-blooded animals. The disease, called protothecosis is manifested by the formation of granulomatous lesions with chronic evolution, localized in different tissues and organs, with systemic forms described the cows (Taniyama et al., 1994) and dogs (Souza et al., 2009). Illness occurs more frequently in patients showing immunodeficiencies or other chronic diseases that are subject to abusive treatment with antibiotics. The greatest risk is observed in dairy cows with mastitis that can evolve as an epidemic outbreak. Following the multiplication and development of granulomas, normal tissue architecture and function is impaired, even affecting vital organs.

As a result of increasing frequency of cases and the antibiotic inefficiency, papers that have described the effect of iodine and iodine based products on Prototheca were published, being underlined their algaecide effect. An in vitro study on 27 P. zopfii strains isolated from cow's milk

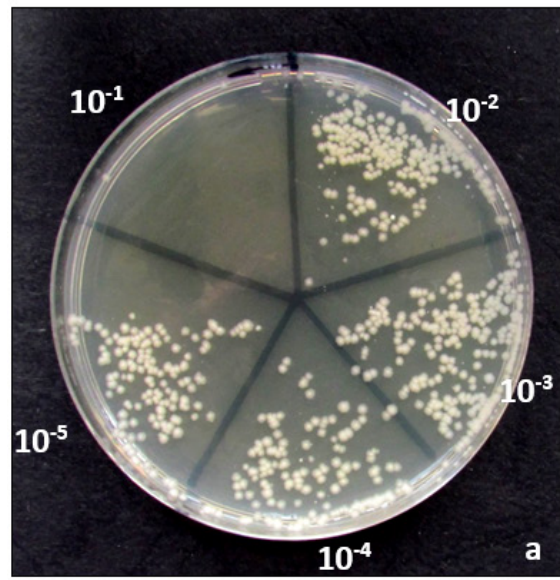

4a) aspect for iodine tincture

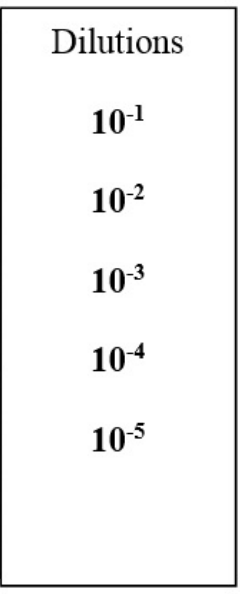

Fig. 4. Prototheca zopfii (strain 22)

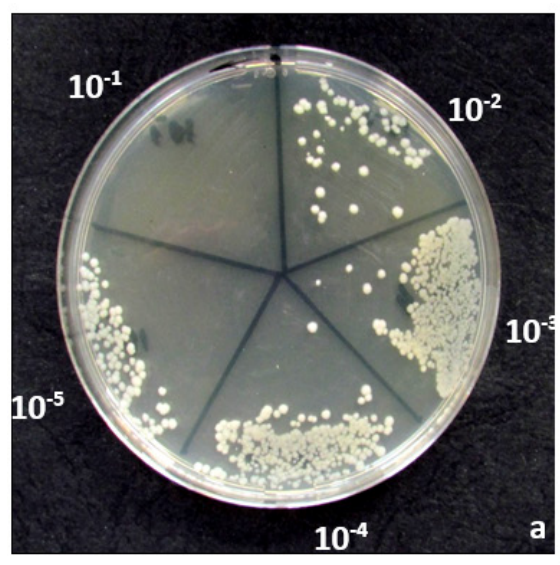

5a) aspect for iodine tincture

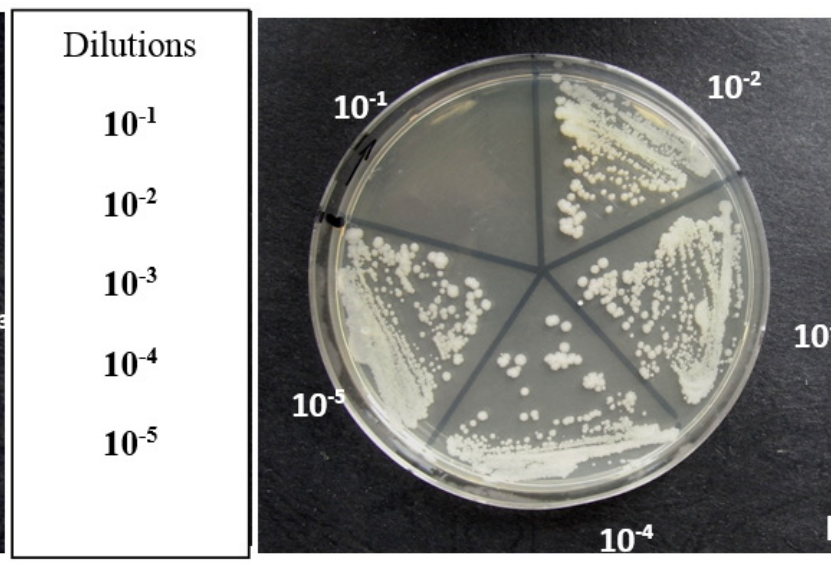

$5 b)$ aspect for betadine

Fig. 5. Prototheca wickerhamii (strain 16529 ATCC) 
shows that iodine preparations showed algaecide effect at low concentrations (0.15652-0.625\%) and recommends its use for routine hygiene (as pre- and post-dipping) in cattle mastitis control (Salerno et al., 2010). The algaecide effect of povidone iodine was also highlighted on $20 \mathrm{P}$. zopfii strains, both biotype 1 (10 strains) and biotype 2 (10 strains) (Sobukawa et al., 2011). In another study on $15 P$. zopfii strains tested for sensitivity to iodine and chlorhexidine using dilution method on Sabouraud dextrose broth, was demonstrated that both drugs have algaecide effect, with minimum microbicide concentration (MMC):0.3125\%-1.25\% for iodine and 0.0048 - $0.0195 \%$ for chlorhexidine (Krukowski et al., 2013).
Iodine and iodine based products (inorganic and organic), having a high antimicrobial potential and broad spectrum, may find use in the treatment of many diseases, including those caused by unicellular algae of the genus Prototheca.

\section{CONCLUSIONS}

By agar diffusimetrical technique it was established that all tested Prototheca strains were sensitive to iodine products, the induced effect being algaecide.

Testing on different inoculum densities, allowed to conclude that the inhibitory effect occurred regardless of density, with small differences in the size of the inhibition zones.
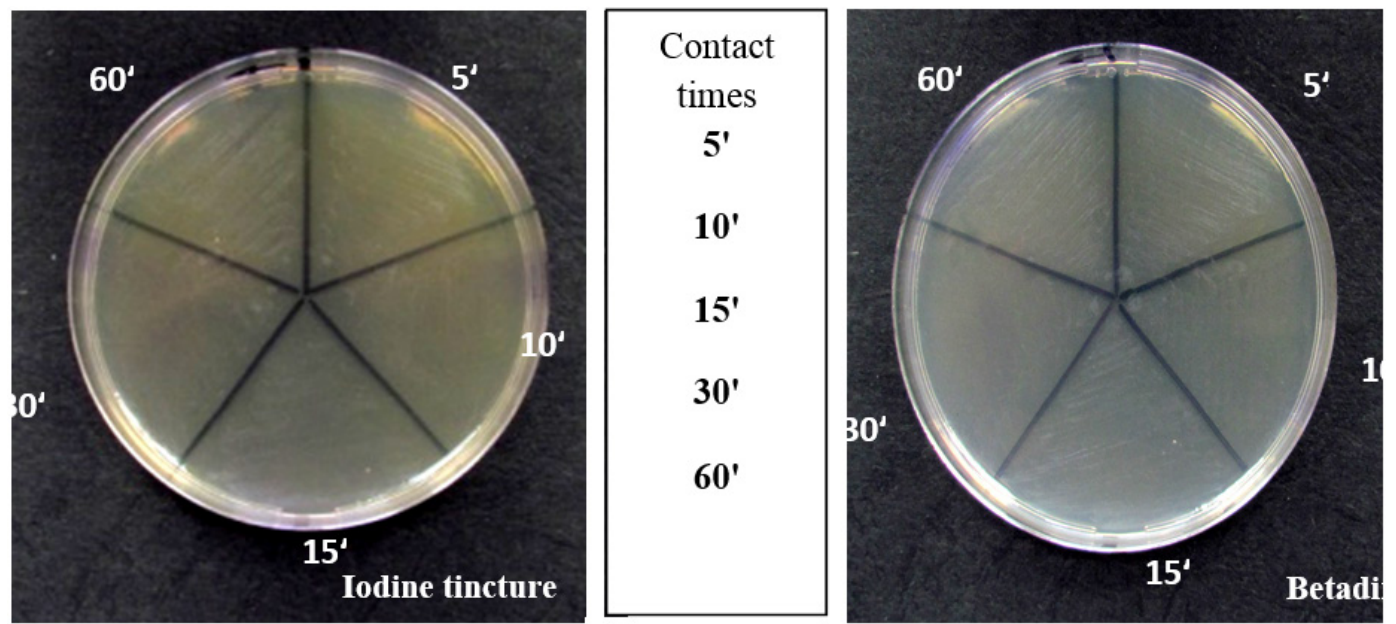

Fig. 6. Prototheca wickerhamii (strain 16529 ATCC) algaecide effect for different contact times (no colonies developed)
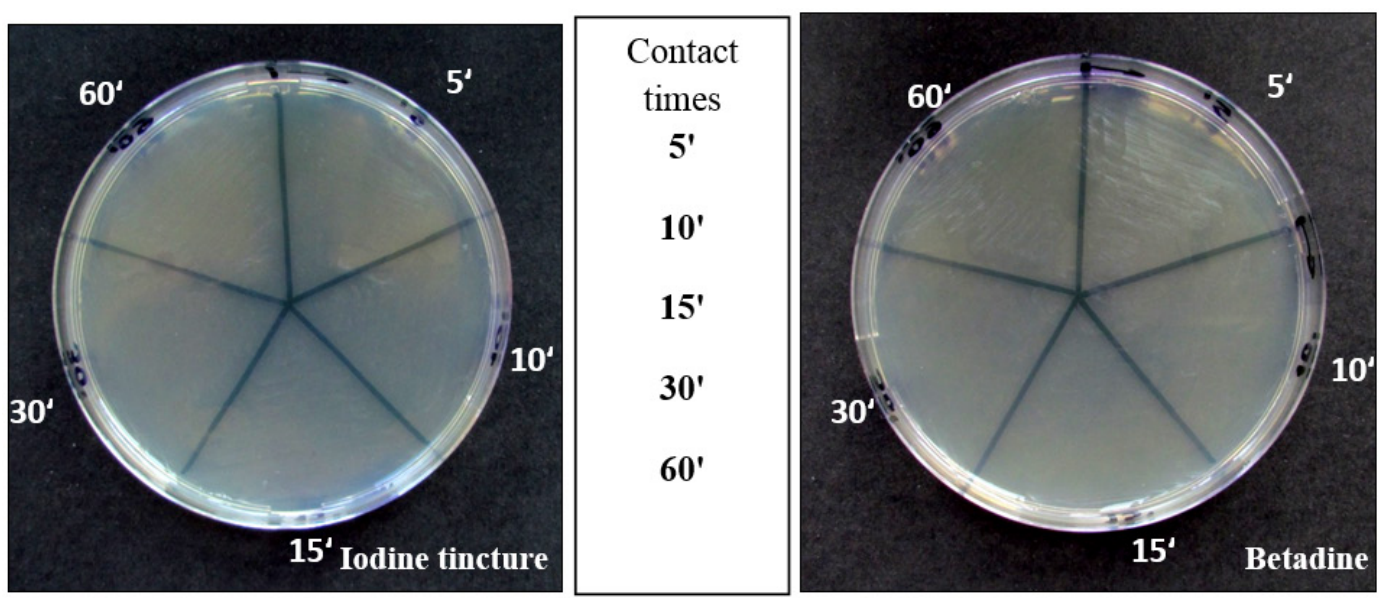

Fig. 7. Prototheca zopfii (strain 22)

algaecide effect for different contact times (no colonies developed) 
Algaecide effect was also demonstrated by dilution method in liquid culture medium (dilution rate $\mathrm{x} 10$ ), stating that it occurred only at $10^{-1}$ dilution, and never produced at higher dilutions, respectively $10^{-2}$ to $10^{-5}$.

In relation to the contact time, set for iodine tincture and betadine, it can be shown that the algaecide effect is fast, within 5 min of contact.

\section{REFERENCES}

1. Azizoglu RO, Lyman R, Anderson KL (2013). Bovine Staphylococcus aureus: dose response to iodine and chlorhexidine and effect of iodine challenge on antibiotic susceptibility. J Dairy Sci 96(2):993-999.

2. Burck RI (1988). Povidone-iodine solution in wound treatment. Phys Ther 78(2):212-8.

3. Buyukmihci N, Rubi LF, DePaoli A (1975). Protothecosis with ocular involvement in a dog. JAVMA 167(2):158-161.

4. Cooper R (2004). A review of the evidence for the use to topical antimicrobial agents in wound care. World Wide Wounds, Centre for Biomedical Sciences School od Applied Sciences, University of Welles Institute Cardiff. http://worldwidewounds.com/2004/february/Cooper/ Topical-Antimicrobial-Agents.html

5. Drosou A, Falabella A, Krisner RS (2003). Antiseptics on wounds: an area of controversy. Wounds 15(5):149166.

6. El Behiry A, Schlenker G, Szabo I, Roesler U (2012). In vitro susceptibility of Staphylococcus aureus strains isolated from cows with subclinical mastitis to different antimicrobial agents. J Vet Sci 13(2):153-161.

7. Giacometti A, Cirioni O, Greganti G, Fineo A, Ghiselli R, Del Pretem S, et al (2002). Antiseptic compounds still active against bacterial strains isolated from still surgical wound infectious increasing antibiotic resistance. Eur. J Clin Microbiol Infect Dis 21(7):553-556.

8. Gottardi W (1999). Iodine and disinfection: theoretical study on mode de action, efficiency, stability, and analytical aspects in the aqueous system. Arch Pharm (Weinheim) 332:151- 157

9. Krukowski H, Lisowski A, Nowakowicz-Debek B, Wlazło L (2013). Susceptibility of Prototheca zopfii strains isolated from cows mastitis to chlorhexidine and iodine. Turk J Vet Anim Sci 37:106-108.

10. Lacey RW, Catto A (1993). Action of povidone-iodine against methicilin-sensitive and - resistant cultures of Staphylococcus aureus. Postgrad Med J 69(3):S78-83.

11. Lamme EN, Gustafsson TO, Middelkoop E (1998). Cadexomer-iodine ointment show stimulation of epidermal regeneration in experimental full-thickness wounds. Arch Dermatol Res 290(1-2):18-24.
12. McLure AR, Gordon J (1992). In vitro evaluation of povidone-iodine and chlorhexidine against methicillin resistance Staphylococcus aureus. J Hosp Infect 21(4):291294.

13. Mertz PM, Oliviera-Gandia MF, Davis SC (1999). The evaluation of a cadexomer iodine wound dressing on methicillin resistance (MRSA) in acute wounds. Dermatol Surg 25(2):89-93.

14. Phillips PL, Wolcott RD, Fletcher J, Schultz GS (2010). Biofilms Made Easy. Wounds International 1(13). Avaiable from: http://www.woundsinternational.com.

15. Răpuntean S (2002). Studiu asupra algelor unicelulare din genul Prototheca: aspect epidemiologice, morfoculturale, biochimice, antigenice și semnificația etiopatogenetică. Teză de doctorat, USAMV, Cluj-Napoca.

16. Salerno T, Ribeiro MG, Lagnoni H, Siqueira AK, Costa E, Melville PA, Bueno VF, Yamamura AA Roesler U, daSilva AV (2010). In vitro algaecide effect of sodium hypochlorite and iodine based antiseptics of Prototheca zopfii strains isolated from bovine milk. Res Vet Sci 88(2):211-213.

17. Sibbald RG, Leaper DJ, Queen D (2011). Iodine Made Easy. Wounds International 2(2):1- 4.

18. Sobukawa H, Watanabe $M$, Kano R, Ito T, Onozaki M, Hasegawa A, Kamata H (2011). In vitro Algaecide Effect of Disinfectants on Prototheca Genotypes 1 and 2. J Vet Med Sci 73(11):1527-1599.

19. Souza NL, Estrela-Lima A, Moreira LTE, Ribeiro LGR et al (2009). Systemic canine protothecosis. Braz J Vet Pathol 2(2):102-106.

20. Taniyama H, Okamoto F, Kurosawa T, Furuoka H, Kaji Y, Okada H, Matsukawa K (1994). Diseminated Protothecosis caused by Prototheca zopfii in a cow. Vet Path 31(1): 123- 126.

21. Wirth FA, Passalacqua JA, Kao G (1999). Disseminated cutaneous protothecosis in an immunocompromised host: a case report and literature rewiev. Cutis 63(3):185188.

22. Woolorich A, Koestenblatt E, Don P, Szaniawski W (1994). Cutaneous prototecosis and AIDS. J Amer Acad Dermatol 31(5/II):920-924.

23. ***The End of Antibiotics and the Rise of Iodine as an Effective Alternative. http://www.naturalnews. com/022800_antibiotic_infection.htm

24. ${ }^{* * *}$ Iod. Wikipedia. http://en.wikipedia.org/wiki/iodine.

25. ${ }^{* * *}$ Iodine Disinfection in the use of individual Water Purification Devices. www.phc.amedd.army.mil/

26. ${ }^{* * *}$ Clinical and Laboratory Standard Institute (2013). Performance Standards for Antimicrobial Disk and Dilution Susceptibility Test for Bacteria Isolated from Animals. Vet 01-A4. 